\title{
Gain functionalization of silica microresonators
}

\author{
Lan Yang and K. J. Vahala \\ Department of Applied Physics, California Institute of Technology, Pasadena, California 91125
}

Received November 20, 2002

\begin{abstract}
Erbium-doped solgel films are applied to the surface of silica microspheres to create low-threshold microcavity lasers. This gain functionalization can be applied by use of a number of different dopants, thereby extending the wavelength range of this class of device. Also, by varying the doping concentration and thickness of the applied solgel layer, one can vary the laser dynamics so that both continuous-wave and pulsating modes of operation are possible. (C) 2003 Optical Society of America

OCIS codes: $140.3500,140.4780,060.2380,160.5690$.
\end{abstract}

Microcavities formed by surface tension (droplets and solid spheres or spheroids) can exhibit quality factors in excess of $1 \times 10^{9}$ and are of interest in cavity QED, nonlinear optics, photonics, and sensing. When silica microspheres are doped with any number of rare-earth ions, ultralow-threshold microlasers are possible. ${ }^{1-3}$ The preparation of these devices requires acquisition of bulk samples of rare-earth-doped glass that are subsequently processed into a spherical cavity. Here, an alternative to this process is presented in which ready-made microspheres of undoped silica serve as a base resonator structure and gain functionalization of the surface is performed with an erbium-doped solgel film (see Fig. 1). Solgel films are readily doped with a number of different rare-earth ions, ${ }^{4-6}$ as well as other materials, ${ }^{7,8}$ thereby making this a more versatile method for preparation of active microspheres. In addition, the solgel preparation process allows for precise control of dopant concentration, making possible the study of a range of inversion concentrations. Finally, it is shown below that the thin-film nature of the gain layer has an important effect on laser dynamics.

We prepared the solgel starting solution by hydrolyzing tetraethoxysilane (TEOS) in water under acid conditions $(\mathrm{pH}, \sim 1)$ with isopropanol as the cosolvent. Erbium ions were introduced by addition of $\mathrm{ErNO}_{3} \cdot 5 \mathrm{H}_{2} \mathrm{O}$ with a weight ratio of $\mathrm{ErNO}_{3} \cdot 5 \mathrm{H}_{2} \mathrm{O} / \mathrm{TEOS} \sim 0.2$ wt. $\%{ }^{9}$ The mixture was then stirred vigorously at $70^{\circ} \mathrm{C}$ for $10 \mathrm{~h}$. After aging the sol solution at room temperature for another $10 \mathrm{~h}$, we immersed silica microspheres in the solution. We formed the initial pure-silica microspheres by heating the end of a tapered fiber tip with a $\mathrm{CO}_{2}$ laser as described by Knight et al. ${ }^{10}$ Multiple process cycles were used to build up a desired layer thickness. Each process cycle consisted of dipping the sphere in the solgel solution for $20 \mathrm{~min}$, followed by heating it in an oven at $160{ }^{\circ} \mathrm{C}$ for $10 \mathrm{~min}$. The layer thickness obtained in a processing cycle depended on the dipping time and solution viscosity. In this experiment the buildup rate was estimated to be $\sim 0.3 \mu \mathrm{m} /$ cycle (determined by observation of layer thickness after multiple cycles). Every two cycles, the spheres were irradiated with the $\mathrm{CO}_{2}$ laser for several seconds. The laser intensity was sufficient to induce flow and densification of the solgel layer. In addition, microcracking that was present in the solgel surface was annealed out by this process. By repeating this process, we varied the coating thickness. Silica spheres ranged in diameter from 50 to $80 \mu \mathrm{m}$, and the coating thickness was varied from one to tens of micrometers. The $\mathrm{Er}^{3+}$-doping concentration of the resulting doped shell was estimated to be $\sim 10^{19} \mathrm{~cm}^{-3}$.

Optical coupling to the spherical microcavities both for pumping and for laser output extraction was performed with optical fiber tapers. Taper coupling was used previously to demonstrate microsphere lasers in the telecommunication band. ${ }^{2}$ It makes possible the resonant excitation of specific whispering-gallery modes (WGMs) and efficient pumping of a small gain volume within the sphere. The typical waist diameter of the tapers used to couple pump power and collect laser emission was $\sim 1.6 \mu \mathrm{m}$.

Whispering-gallery mode (WGM) resonances correspond to light trapped in circulating orbits just within the surface of the spheroidal particle. ${ }^{11,12}$ The modal indices are similar to those used to characterize simple atomic systems with radial $(n)$, orbital $(l)$, azimuthal $(m)$, and polarization (TE or TM) indices needed to specify a mode completely. In particular, the angular distribution of the modes is given by the spherical harmonics $Y_{l m}(\theta, \varphi)$ (see Fig. 2), and the WGM modes with best spatial overlap to the fiber taper have their power concentrated near the equatorial plane $(m \approx l)$ with a low radial coordinate, $n \approx 1$. These same modes are also best able to pump the active medium's surface layer over a radial thickness given approximately by the material wavelength of the pump

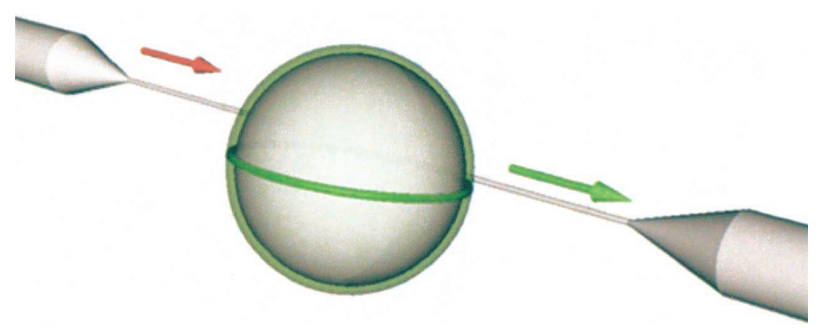

Fig. 1. Schematic of a thin-film-coated microsphere laser coupled with a fiber taper. The red and green arrows represent input pump and output lasing waves, respectively. 

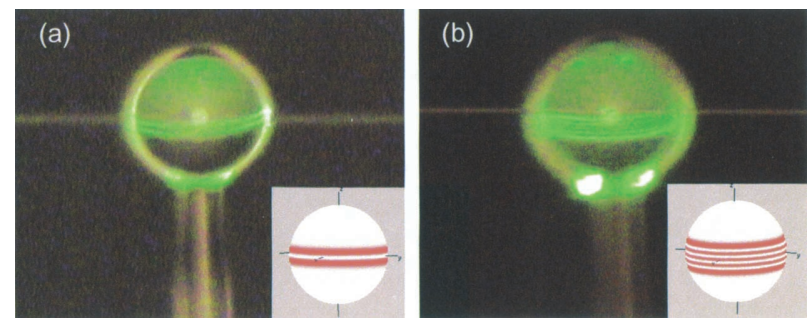

Fig. 2. Images of the WGMs in the taper-sphere coupling zone: (a) $l-m=1$, (b) $l-m=4$. The green rings are upconverted photoluminescence. The inset shows spherical harmonics ${ }^{13} Y_{l m}(\theta, \varphi)$ for (a) $l-m=1$ and (b) $l-m=4$.

band. The pumped region will overlap the emission band modes, making possible lasing action. For a surface-layer thickness somewhat less than the radial width of the pump mode, inversion is expected within annular-shaped equatorial bands. Conversely, when the thickness is substantially greater than the pump-mode radial width, there will remain unpumped regions that can provide saturable absorption to the longer-wavelength lasing modes. In addition to modifying the threshold characteristics, saturable absorption is known to modify lasing dynamics such that pulse behavior is possible. This distinction between thick and thin shell behavior is investigated below.

The pump wave was in the 980-nm wavelength band and was provided by a tunable single frequency, narrow-linewidth $(<300-\mathrm{kHz})$ external-cavity laser. We scanned the pump wavelength initially to survey pumping modes. These were observable from monitoring the transmission versus tuning and also by use of a camera to monitor green excited-state emission from the sphere as the pump laser tuned into resonance with various pump modes. Figure 2 shows representative lateral emission distributions observed for different WGMs in the sphere-taper coupling zones. The pump power coupled to the sphere was measured as the difference of the launch power into the taper and the transmitted power after the taper. Both continuous-wave (cw) operation and pulse-mode operation were possible by control of the solgel coating thickness. cw laser operation was observed with a coating thickness of roughly $1 \mu \mathrm{m}$, whereas pulsation mode laser operation was possible for coatings $\gtrsim 5 \mu \mathrm{m}$ in thickness. The thickness of the doped layer was estimated by observation of the thickness of the sphere both before and after the coating process.

A typical laser spectrum for cw operation is presented in the inset of Fig. 3. This was measured with an optical spectrum analyzer with a resolution bandwidth setting of $0.5 \mathrm{~nm}$. Multiline operation was also observed and depended on the pump wavelength selected; however, by tuning of the pump wave it was always possible to achieve single-line operation. ${ }^{14}$ This is believed to result from the strong spatial-mode selection that is possible when the so-called fundamental WGM (equatorial ring orbit) is resonantly pumped. Figure 3 shows the laser output versus the pump power absorbed by the microsphere for $\mathrm{cw}$ operation. The threshold was estimated to be $\sim 28 \mu \mathrm{W}$, and the laser reached an output power of $6 \mu \mathrm{W}$. Above threshold, the laser output power varied linearly with absorbed pump power. A laser output power of up to $10 \mu \mathrm{W}$ was observed for single-mode pm operation. For convenience during the experiments, the sphere was in contact with the taper (i.e., there was zero air gap). This greatly restricted control of coupling and potentially limited the optimization of laser output power. In addition, optimal coupling of the pump requires balancing of taper loading with round-trip loss (dominated by erbium absorption in the shell layer). Optimal coupling of laser emission also requires optimization of loading, but not necessarily for the same conditions as for the pump. Other factors affecting coupling are phase matching and field overlap between the taper and sphere modes in both the pump band and the emission band.

Figure 4 shows the measured pulse frequency versus the square root of the laser output power for pulsation mode operation. The frequency was in a range from tens of kilohertz to several hundred kilohertz. An electrical spectrum analyzer was employed for this measurement. The observed linear behavior is consistent with undamped relaxation oscillations. ${ }^{15}$ The ability to induce pulsation mode operation by control of the shell thickness is attributed to unpumped inner regions of the shell that can provide saturable absorption to the lasing mode. This conjecture is consistent with the observation of pm operation in previous microsphere laser work using bulk-doped glass for sphere fabrication. ${ }^{2}$ Azimuthal surface regions can also potentially provide saturable absorption. However, if they were present here, they were alone insufficient to induce instability.

In conclusion, we have demonstrated the gain functionalization of silica microspheres by use of doped solgel films. This technique provides a way to achieve a range of possible gain media in the microsphere system. Likewise, other possible surface layers that target applications such as nonlinear optics in a microcavity ${ }^{16}$ may also benefit from this approach.

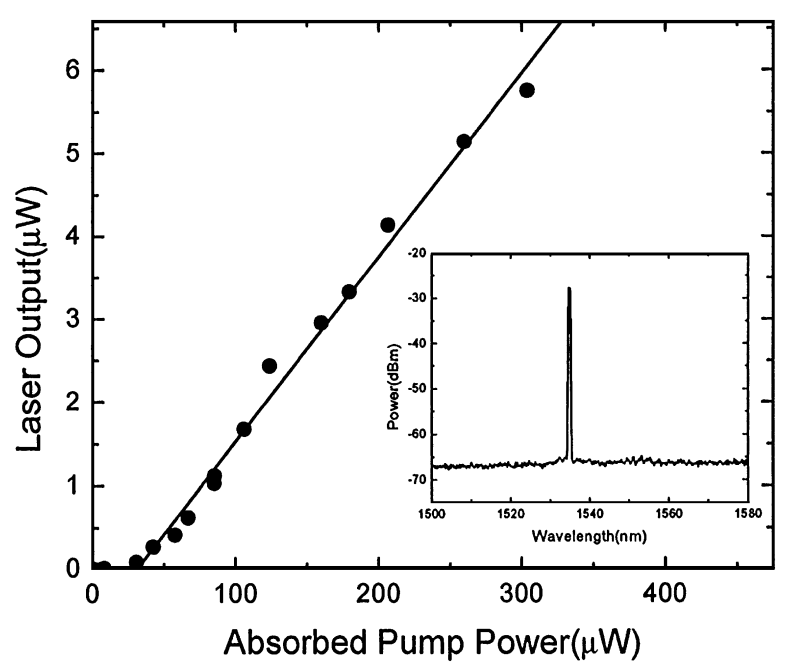

Fig. 3. Laser output power versus absorbed pump power in the microsphere laser. The inset shows the emission spectrum of the microsphere laser. 


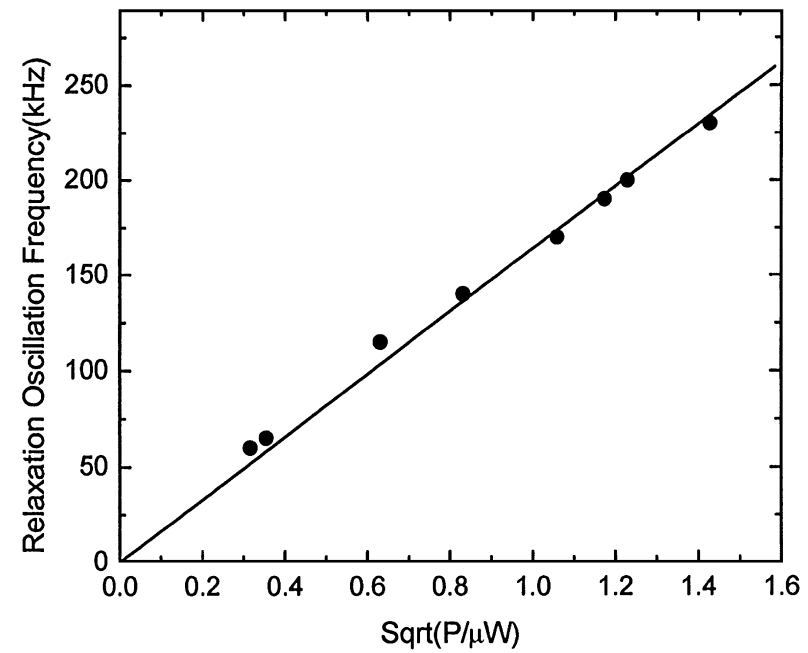

Fig. 4 Measured pulse frequency versus the square root of the laser output power, $P$.

An important feature of a solgel gain layer is the ability to quench previously observed pulses in these devices, thereby yielding cw laser operation. For a shell thickness of roughly $1 \mu \mathrm{m}$, cw laser operation was observed. This behavior as well as the onset of pulses for thicker active shells is attributed to unpumped and hence saturable absorbing regions that can be present in thick shells.

This work was supported by the Defense Advanced Research Project Agency, the National Science Foundation, and the Caltech Lee Center. We gratefully thank Ming Cai and Sean S. Spillane for helpful discussion. K. J. Vahala's e-mail address is vahala@its.caltech.edu.

\section{References}

1. V. Sandoghdar, F. Treussart, J. Hare, V. Lefevre-Seguin, J. M. Raimond, and S. Haroch, Phys. Rev. A 54, 1777 (1996).

2. M. Cai, O. Painter, K. J. Vahala, and P. C. Sercel, Opt. Lett. 25, 1430 (2000).

3. W. von Klitzing, E. Jahier, R. Long, F. Lissillour, V. Lefevre-Seguin, J. Hare, J. M. Raimond, and S. Haroche, Electron. Lett. 35, 1745 (1999).

4. X. Orignac, D. Barbier, X. M. Du, and R. M. Almeida, Appl. Phys. Lett. 69, 895 (1996).

5. S. Bruynooghe, A. Chabli, F. Bertin, F. Pierre, and G. Leflem, J. Mater. Res. 12, 2779 (1997).

6. S.-Y. Chen, C.-C. Ting, and C.-H. Li, J. Mater. Chem. 12, 1118 (2002).

7. A. Arena, S. Patane, G. Saitta, G. Rizzo, S. Galvagno, and G. Neri, J. Mater. Res. 17, 2095 (2002).

8. W. T. Dong and C. S. Zhu, Mater. Lett. 45, 336 (2000).

9. L. H. Slooff, M. J. A. de Dood, A. van Blaaderen, and A. Polman, J. Non-Cryst. Solids 296, 158 (2001).

10. J. C. Knight, G. Cheung, F. Jacques, and T. A. Birks, Opt. Lett. 22, 1129 (1997).

11. R. K. Chang and A. J. Campillo, Optical Processes in Microcavities (World Scientific, Singapore, 1996).

12. M. L. Gorodetsky and V. S. Ilchenko, Opt. Commun. 113, 133 (1994).

13. B. P. Reid, http://www.bpreid.com/applets/poasDemo. html.

14. Within the observable resolution limit of the optical spectrum analyzer.

15. A. E. Siegman, Lasers (University Science, Mill Valley, Calif., 1986).

16. S. Spillane, T. Kippenberg, and K. Vahala, Nature 415, 621 (2002). 